\title{
Salud Colectiva: informe de gestión editorial 2010
}

\section{Salud Colectiva: report on editorial management 2010}

\author{
Martinovich, Viviana'; Di Cecco, Vanessa² \\ 1 Editora Ejecutiva, Salud Colectiva. Instituto de Salud Colectiva, Universidad Nacional de Lanús, Argentina. \\ vivianamartinovich@gmail.com \\ ${ }^{2}$ Editora Asociada, Salud Colectiva. Instituto de Salud Colectiva, Universidad Nacional de Lanús, Argentina. \\ vanessa.dicecco@gmail.com
}

A partir de este año, hemos decidido comenzar a publicar algunos datos e indicadores sobre la actividad editorial de Salud Colectiva, vinculados con los procesos de evaluación y publicación. Somos conscientes de que las cifras reflejan los primeros pasos de una revista que en 2010 cumplía solo 6 años, en los que atravesó por las dificultades propias de los proyectos editoriales que no están sustentados por lógicas económicas e industriales, sino construidos a partir de la necesidad de sostener un espacio de reflexión y compromiso con la realidad que los rodea. Entendemos que este tipo de propuestas editoriales tienen procesos propios de crecimiento y de legitimación que se deberían comenzar a revalorizar y analizar con criterios acordes a su lógica de producción. Por lo tanto, publicar estos indicadores tiene como fin situarnos en un escenario y en un tiempo específico para comenzar a contar nuestra historia desde sus orígenes, lo que nos permitirá a futuro poder analizar el camino transitado y visualizar el proceso como una construcción diacrónica.

En esta oportunidad, presentamos una selección de los datos generados por dos de los cuatro módulos de gestión editorial que actualmente utiliza la revista: el módulo de "evaluación" y el de "publicación". El primero, en el que se realiza el seguimiento y control de los procesos de revisión de los documentos recibidos, permite conocer el origen geográfico de las contribuciones, los porcentajes de artículos aceptados y rechazados, quiénes participaron de esas evaluaciones, etc. El segundo módulo, en el que se gestiona todo el proceso de edición de los trabajos aprobados, proporciona información acerca del total de autores, tipo de material publicado, entre otros datos.

\section{MÓDULO EVALUACIÓN}

En el año 2010 recibimos 56 contribuciones, de las cuales 19 (33,93\%) fueron enviadas por autores de instituciones argentinas y 37 $(66,07 \%)$ por autores de instituciones de otros países (a) (Figura 1). El alto porcentaje de trabajos enviados desde el exterior refleja la rápida internacionalización de la revista en estos seis años.

De las 56 contribuciones recibidas, el $89,29 \%$ fueron trabajos enviados en forma espontánea para las secciones abiertas a presentación y el 10,71\% corresponden a contribuciones

Figura 1. Porcentajes de artículos recibidos según país de la institución de pertenencia del autor responsable. Año 2010.

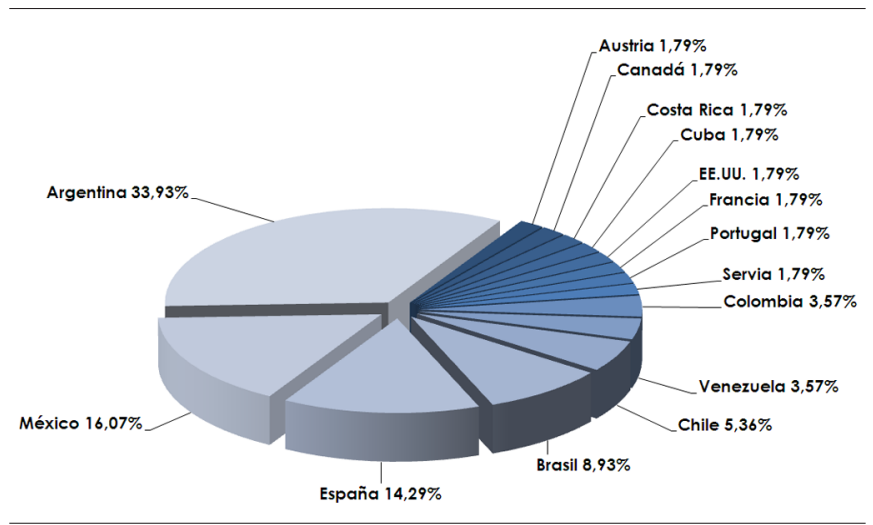

Fuente: Salud Colectiva. 
solicitadas (editoriales) o producidas por el equipo de la revista para otras secciones. Ese 89,29\% de contribuciones no solicitadas, pasó por una primera instancia de preevaluación, cuyo propósito no solo es determinar si el artículo se enmarca en los objetivos y en la política editorial de la revista, sino aunar criterios con los autores sobre el significado y alcance de ciertas prácticas y conceptos vinculados a la publicación de resultados de investigaciones.

Para todos los artículos aceptados en esta primera etapa de preevaluación se abrieron procesos de revisión externa para los que se convocó a un mínimo de dos revisores por artículo. El arbitraje es una tarea honoraria y dar a conocer a quienes aceptaron evaluar los trabajos presentados, es el modo de transmitirles todo nuestro reconocimiento por el tiempo dedicado, el cumplimiento de los plazos y las valiosas opiniones emitidas sobre los textos, muy valoradas además por muchos de los autores. Con todo nuestro agradecimiento, damos a conocer la nómina de aquellos que evaluaron los trabajos presentados en el año 2010 (Cuadro 1), muchos de los cuales realizaron dos y hasta tres revisiones en el transcurso del año.

Como resultado de los procesos de evaluación, el porcentaje de contribuciones aceptadas fue del 41,07\% (Figura 2).

\section{MÓDULO PUBLICACIÓN}

En 2010 se publicaron 24 contribuciones originales e inéditas (b), de las cuales 18 (75\%) corresponden a artículos no solicitados y el $25 \%$ restante a editoriales y producciones propias de la

Figura 2. Porcentajes de contribuciones aceptadas y rechazadas. Año 2010.

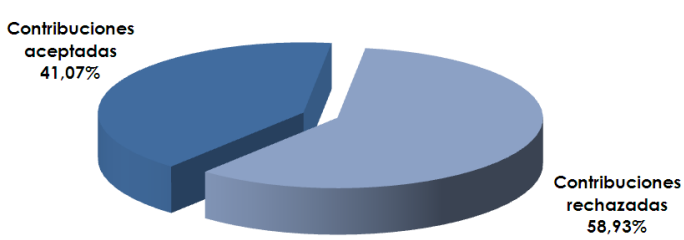

Fuente: Salud Colectiva.
Figura 3. Distribución del total de autores de contribuciones publicadas en Salud Colectiva, según país de la institución de pertenencia. Año 2010.

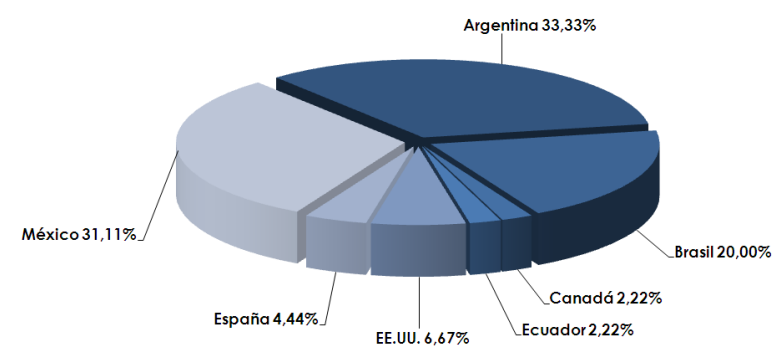

Fuente: Salud Colectiva.

revista para las secciones "Diálogos", "Memoria e Historia" y "Noticias SC".

En las 24 contribuciones publicadas participaron un total de 45 autores: el 33,33\% perteneciente a instituciones nacionales y un $66,67 \%$ a instituciones de otros países, sobre todo de México y Brasil (Figura 3).

La incorporación de nuevos editores asociados, además de asistir tanto en la preevaluación de los trabajos presentados como en el seguimiento de las evaluaciones externas por áreas temáticas, permitió la creación de secciones como "Memoria e Historia" que reunió a dos personalidades como Mario Testa y Jairnilson Silva Paim con el propósito de recuperar la memoria del campo de la medicina social y la salud colectiva desarrolladas desde los años 60 en América Latina, y la sección "Diálogos" en la que Cristian Alarcón y Philippe Bourgois reflexionaron sobre sus obras y los significados de las formas de producir un relato sobre el narcotráfico. Consideramos que la capacidad de convocar a estas figuras y producir estas secciones también es un índice de nuestro crecimiento como revista.

Empezar a publicar estos datos es una manera de compartir los procesos editoriales que no solo reflejan el esfuerzo mancomunado de todo el equipo editorial, sino que son producto también de la colaboración y participación activa de los lectores, autores y revisores y del apoyo institucional de la Universidad Nacional de Lanús. Seguimos juntos en un camino de valorización de la producción científica y de la práctica editorial latinoamericanas. 
Cuadro 1. Nómina de revisores externos que evaluaron artículos ingresados en 2010.

- Abdala, Félix - Centro de Estudios de Población. Argentina

- Aguirre, Patricia - Universidad Nacional de San Martín. Argentina

- Amable, Marcelo - Universidad Nacional de Lanús. Argentina

- Ase, Iván - Universidad Nacional de Córdoba. Argentina

- Barata, Rita - Universidade de São Paulo. Brasil

- Borrell, Carme - Agència de Salut Pública de Barcelona. España

- Botazzo, Carlos - Universidade de São Paulo. Brasil

- Brissón, Maria Eugenia - Universidad Nacional de Lanús. Argentina

- Cardaci, Dora - Universidad Autónoma Metropolitana-Xochimilco. México

- Castellanos, Pedro Luis - IDESARROLLO. República Dominicana

- Castiel, Luis - Escola Nacional de Saúde Pública. Brasil

- Castronovo, Raquel - Universidad Nacional de Lanús. Argentina

- Checa, Susana - Universidad de Buenos Aires. Argentina

- Chiara, Magdalena - Universidad Nacional de General Sarmiento. Argentina

- Chorny, Adolfo - Escola Nacional de Saúde Pública. Brasil

- Comelles, Josep M. - Universitat Rovira i Virgili, Tarragona. España

- De Maio, Fernando - University Simon Fraser. Canadá

- Deslandes, Suely - Escola Nacional de Saúde Pública. Brasil

- Domínguez Mon, Ana - Universidad de Buenos Aires. Argentina

- Donalisio, María Rita - Universidade Estadual de Campinas. Brasil

- Drinot, Paulo - University of Manchester. Reino Unido

- Duek, María Celia - Universidad Nacional de Cuyo. Argentina

- Escudero, José Carlos - Universidad Nacional de Luján. Argentina

- Facchini, Luiz Augusto - Universidade Federal de Pelotas. Brasil

- Gomes, Romeu - Escola Nacional de Saúde Pública. Brasil

- Guevel, Carlos - Universidad Nacional de Lanús. Argentina
- Hennington, Élida - Escola Nacional de Saúde Pública. Brasil

- Homedes, Núria - University of Texas. EE.UU.

- Iriart, Jorge - Universidade Federal de Bahia. Brasil

- López Arellano, Oliva - Universidad Autónoma Metropolitana-Xochimilco. México

- López, Elsa - Universidad de Buenos Aires. Argentina

- Macías, Guillermo - Universidad Nacional de Lanús. Argentina

- Márquez Valderrama, Jorge - Universidad Nacional de Colombia. Colombia

- Martín, Ana Laura - Universidad Nacional de Lanús. Argentina

- Mercado, Francisco - Universidad de Guadalajara. México

- Módena, Allegroni María Eugenia - CIESAS. México

- Neffa, Julio César - CEIL-PIETTE, CONICET. Argentina

- Osorio Carranza, Rosa María - CIESAS. México

- Ozollo, Javier - Universidad Nacional de Cuyo. Argentina

- Palmer Steven - University of Windsor. Canadá

- Pecheny Mario - Universidad de Buenos Aires. Argentina

- Pedersen, Duncan - McGill University. Canadá

- Raus, Diego Martín - Universidad Nacional de Lanús. Argentina

- Recoder, Laura - Universidad Nacional de Lanús. Argentina

- Romani, Oriol - Universitat Rovira i Virgili, Tarragona. España

- Sacchi, Mónica - Universidad Nacional de Salta. Argentina

- Salas-Valenzuela, Monserrat - Instituto Nacional de Ciencias Médicas y Nutrición. México

- Silva Ayçaguer, Luis Carlos - Centro Nacional de Información de Ciencias Médicas. Cuba

- Silva Paim, Jairnilson - Universidade Federal de Bahia. Brasil

- Teixeira, Ricardo - Universidade de São Paulo. Brasil

- Ugalde, Antonio - University of Texas. EE.UU.

- Vázquez, Enrique - Organización Panamericana de la Salud. Paraguay

- Vega López, María Guadalupe - Universidad de Guadalajara. México

- Ynoub, Roxana - Universidad de Buenos Aires. Argentina 


\section{NOTAS FINALES}

a. En el módulo de evaluación, el origen geográfico de las contribuciones se define por la institución de pertenencia del autor responsable de la correspondencia. b. Las colaboraciones publicadas en 2010 no se condicen necesariamente con las colaboraciones recibidas y evaluadas en 2010, dado que algunas de ellas fueron enviadas en 2009 y aprobadas para su publicación en 2010.

\section{FORMA DE CITAR}

Martinovich V, Di Cecco V. Salud Colectiva: informe de gestión editorial 2010. [Noticias SC]. Salud Colectiva. 2011;7(3):401-404. 